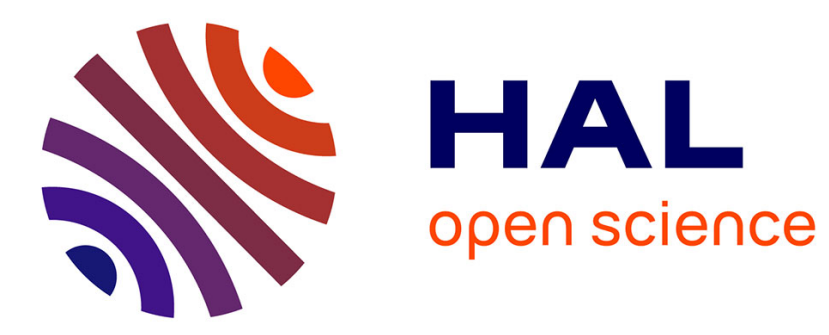

\title{
Forces and charges on an undeformable droplet in the DC field of a plate condenser
}

\author{
Jean-Maxime Roux, Jean-Luc Achard, Yves Fouillet
}

\section{To cite this version:}

Jean-Maxime Roux, Jean-Luc Achard, Yves Fouillet. Forces and charges on an undeformable droplet in the DC field of a plate condenser. Journal of Electrostatics, 2008, 66 (5-6), pp.283-293. 10.1016/j.elstat.2008.01.008 . hal-00267652

\section{HAL Id: hal-00267652 \\ https://hal.science/hal-00267652}

Submitted on 26 Feb 2020

HAL is a multi-disciplinary open access archive for the deposit and dissemination of scientific research documents, whether they are published or not. The documents may come from teaching and research institutions in France or abroad, or from public or private research centers.
L'archive ouverte pluridisciplinaire HAL, est destinée au dépôt et à la diffusion de documents scientifiques de niveau recherche, publiés ou non, émanant des établissements d'enseignement et de recherche français ou étrangers, des laboratoires publics ou privés. 


\title{
Forces and charges on an undeformable droplet in the DC field of a plate condenser
}

\author{
J.M. Roux ${ }^{\mathrm{a}, *}$, J.L. Achard ${ }^{\mathrm{b}}$, Y. Fouillet ${ }^{\mathrm{a}}$ \\ ${ }^{a}$ Department of Technology for Biology and Health, CEA/LETI, 17 av. des Martyrs, 38054 Grenoble Cedex 9, France \\ ${ }^{\mathrm{b}}$ Laboratoire des Ecoulements Géophysiques et Industriels, 38041 Grenoble Cedex 9, France
}

A single conducting drop resting on the lower plane electrode of a horizontal condenser and surrounded by a dielectric fluid is considered. When a DC field is applied to the electrodes, the drop acquires electric charges and is subjected to an electrostatic force normal to the electrode. This driving force may eventually detach the drop if the applied field strength exceeds a threshold value.

For small drops, the gravitational field and the electrical force effect can be neglected with respect to the surface tension. In this case, it may be assumed that drops are undeformable and keep a spherical cap shape. Based on this model, charges and forces are calculated analytically in any wetting conditions. To this end, previous studies concerning solid spheres are conveniently extended.

The usefulness of the above model is then considered to determine the lift-off threshold value. For non-wetting conditions, new experimental results are presented: they fit precisely the derived theoretical lift-off conditions. For wetting conditions, the preceding calculations are shown to be unable to provide any testable criterion. The undeformability assumption has to be relaxed.

\section{Introduction}

A conducting droplet immersed in a dielectric medium and set on the lower plate of a condenser acquires an electrical charge when subjected to a uniform electrical field. Above an electrical field threshold value the electrical force lifts the liquid particle. After lift-off, the droplet adopts the back and forth movement previously described by several authors for solid particles [1,2]. While solid particles are easier to study, droplets have a more complex behavior due to the possible electrode wetting and surface deformation.

Recently, Khayari et al. [3] have studied experimentally the deformation of a drop subjected to an electric field and have proposed some estimate of the critical electrical field needed to lift the drop, including the adhesion force which tends to retain the drop in contact with the electrode. It is, to our knowledge, the first attempt to describe the complex

\footnotetext{
*Corresponding author.

E-mail address: rouxjm@chartreuse.cea.fr (J.M. Roux).
}

behavior of a droplet in such a situation. They have introduced in their analysis two simplifying assumptions. First, in order to have a first appraisal of the charge acquired by the drop on an electrode they used results obtained for solid conducting sphere (the Maxwell's charge). Moreover, they consider the electrical force to be solely a Coulombic force, neglecting the nearest electrode influence. Lebedev and Skal'skaya [4], Félici [5] and Jones [6] have showed that the electrical force must also include the interaction force with the droplet image due to the electrode plane. They conclude their study by noticing the fact that a complete theoretical model should take into account the electric field distribution around the exact deformed drop. Our work is an attempt to achieve this model and extend their study.

A given drop departs from a pure spherical shape for two distinct physical reasons. On one side, surface tension tends to give a spherical cap shape to the drop. On another side, surface tension may be unable to maintain spherical the upper part of the cap when drops are not small or more precisely when the ratios of gravity and of imposed 
electrical field to surface tension forces are not both very small. In this case, the shape of a sessile drop in a vertical electric field is determined by two opposite constraints: gravity flattens the droplet while the electrical field elongates it (Fig. 1). In this paper, the two above ratios will be even zero and droplets will have a strict spherical cap shape.

To begin with, in the present article, we will focus on non-wetting conditions. Hydrophobic carbon nanotubes covered electrodes are so hydrophobic that droplets behave as liquid balls on them. Using such a conducting superhydrophobic [7] lower electrode permits to treat droplets as spherical solid spheres placed on an ordinary electrode. Lebedev and Skal'skaya [4] electrostatic model is recalled as a theoretical background. These authors computed analytically the charge $Q$ acquired by the sphere, namely the Maxwell's charge, and the electrical force $\mathbf{F}$ exerted by the electrical field on the sphere. These authors solved the Laplace's equation in an " $R$-separable" coordinates system compatible to the problem. Later Félici [5] and Jones [6] confirmed the charge $Q$ and the force $\mathbf{F}$ expressions of Lebedev and Skal'skaya [4] using the method of images. And numerous experimental studies on conducting solid balls validated the theoretical prediction for the force $\mathbf{F}$ [8]. The main results of their calculations are recalled. They are used to derive the minimal electrical field $E_{\infty}^{\mathrm{c}}$ required to lift a conducting sphere. When the droplet lifts off, the reaction exerted by the plane on the droplet becomes zero in the overall momentum balance which provides then $E_{\infty}^{\mathrm{c}}$. An experimental test of this critical field force is undertaken with non-wetting droplets. It comes as an extension of spherical solid particles lift-off studies.

Next, the previous model valid for spherical droplet tangent to the lift-off electrode is extended to more general wetting conditions, i.e. to spherical cap shape droplets. The method used by Lebedev and Skal'skaya [4], for solving the electrostatic problem of a grounded sphere lying on a plane in an electric field, was generalized to the case of a portion of sphere protruding from a plane electrode by Le Ny [9]. This latter gave the charge and force evolutions as functions of the protrusion radii. To some extent, this theoretical study is close to the problem considered in the present paper. By adapting Le Ny's study, the forces and charges on an undeformable droplet in the DC field of a plate condenser are computed as functions of the contact angle to describe the force and the charge evolutions on wetting droplets.

Finally, the droplet lift-off criterion following these results valid for a spherical cap shaped droplet is examined. Contrary to the non-wetting case, it will be shown that expressing how the droplet/plane interaction force decreases, requires to relax the undeformability assumption. In this context, deducing $E_{\infty}^{\mathrm{c}}$ is no more possible.

\section{Formulation of the general problem}

Our problem is presented here in a general way. A conducting droplet of volume $V$ is deposited with a micropippette on the horizontal surface $S_{\mathrm{e}}$ of the lower electrode of a condenser immersed in a dielectric liquid. It will spread under the combined effect of gravity and the capillary forces at the rim until it reaches an equilibrium position (Fig. 1a). Finally, the drop adopts an axisymmetric shape interface $S_{\mathrm{d}}$, sitting on the surface with a contact angle $\theta_{\mathrm{s}}$ and a contact area $S_{\mathrm{c}}$ of radius $c$. Afterwards, the gradually imposed uniform electrical field $\mathbf{E}_{\infty}$, placed parallel to the direction of gravity $\mathbf{g}$, may modify this shape (Fig. 1b); the process is slow enough to let the drop go through a series of steady states. If the droplet is small enough it may ignore $\mathbf{g}$ and $\mathbf{E}_{\infty}$ and keeps a spherical cap

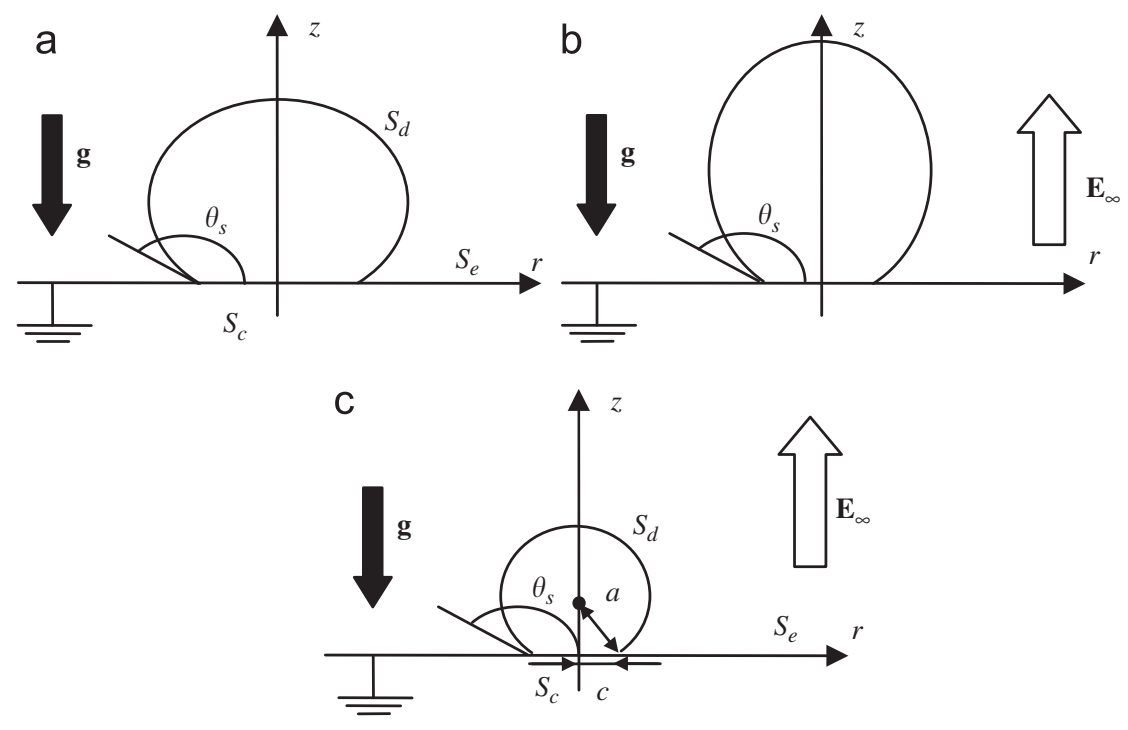

Fig. 1. Schematic description of a droplet subjected to the gravitational field and next to an electrical field. (a) The droplet is flattened by its weight. (b) Afterwards, an electrical field is applied and the droplet is elongated by the electrical force. Within our restrictions the droplet is kept spherical by the surface tension whatever forces act on it (c). 
shape (Fig. 1c) of radius $a$. In any cases, the distance between the two electrodes is large compared to $a_{0}=(3 V / 4 \pi)^{1 / 3}$, radius of the equivalent non-wetting droplet, so that interactions with the upper electrode can be safely neglected. The ratio $\delta=a_{0} / a$ depends on $\theta_{\mathrm{s}}$ via the geometrical relation:

$\delta=\left[\frac{1}{4}\left(2-3 \cos \theta_{\mathrm{s}}+\cos ^{2} \theta_{\mathrm{s}}\right)\right]^{1 / 3}$.

According to the Young-Dupré's equation [10], the contact angle is supposed to be unique. However in our experiments, this angle could not be measured unambiguously because of the, now well known, hysteresis phenomenon [10]. This last effect will be taken into account in a second paper.

\subsection{Electrical equations}

Consider the most general situation shown schematically in Fig. 1a, b. The inner and outer liquids are assumed to be immiscible, incompressible and have no bulk free charges. Subscripts "i", "o", and "s" indicate the variables associated with the inner liquid, outer liquid and the interface $S_{\mathrm{d}}$ itself while the variables without subscripts are applicable to either liquid phases. It is assumed that the electric conductivity $\sigma$ and permittivity $\varepsilon$ of both liquids are uniform. The conductivities ratio is denoted by $\alpha=\sigma_{\mathrm{o}} / \sigma_{\mathrm{i}}$ and the dielectric constants ratio by $\beta=\varepsilon_{\mathrm{o}} / \varepsilon_{\mathrm{i}}$. Both are supposed very small but $\alpha \ll \beta$.

The electrical potential $\varphi$, introduced in the usual way $\mathbf{E}=-\nabla \varphi$, will have to satisfy the Laplace's equation in each phase:

$\Delta \varphi=0$.

The boundary conditions for our configuration are:

(i) At the lower electrode:

$\varphi_{\mathrm{o}}=0 \quad$ on $S_{\mathrm{e}}$

$\varphi_{\mathrm{i}}=0 \quad$ on $S_{\mathrm{c}}$

since the lower electrode defined by the equation $z=0$ is grounded.

(ii) On the fluid/fluid interface:

- The tangential components of the electric field are equal at the interface or equivalently, the electrical potential is continuous across it:

$\varphi_{\mathrm{o}}=\varphi_{\mathrm{i}} \quad$ on $S_{\mathrm{d}}$.

- Admitting there are no steady fluid motions along the interface (which will be proved later), the charge conservation law gives:

$[[\sigma \nabla \varphi \cdot \mathbf{n}]]=0 \quad$ on $S_{\mathrm{d}}$

where the standard bracket notation $[[\mathbf{n} \cdot \mathbf{F}]]=$ $\sum_{\mathrm{k}=\mathrm{i}, \mathrm{o}} \mathbf{n}_{\mathrm{k}} \cdot \mathbf{F}_{\mathrm{k}}$ is used and $\mathbf{n}_{\mathrm{k}}$ is the unit normal vector at the surface pointing outside phase $\mathrm{k}$.
- The Gauss law leads to superficial charges:

$$
[[\varepsilon \nabla \varphi \cdot \mathbf{n}]]=-q_{\mathrm{s}} \quad \text { on } S_{\mathrm{d}} .
$$

(iii) The electric field is finite on the symmetry axis of the drop and the far field tends to the imposed field $\mathbf{E}_{\infty}$ :

$$
\varphi(0, z) \text { is finite on } \mathrm{Oz}
$$

and

(iv)

$\lim _{z \rightarrow \infty} \varphi=-E_{\infty} z$,

where $E_{\infty}$ is the magnitude of $\mathbf{E}_{\infty}$. Birlasekaran [1], studying conducting balls, suggested that the condition (9) is true for a inter-electrode distance $H$ such that $H / 2 a_{0} \gg 3$, where $a_{0}$ is the radius of the ball.

The above equations are non-dimensionalized with lengths measured in units of $a_{0}$, electrical potentials in units of $E_{\infty} a_{0}$ and charges in units of $\varepsilon_{0} E_{\infty} a_{0}^{2}$. For convenience, we do not distinguish dimensionless variables notations. Since the inner fluid conductivity $\sigma_{\mathrm{i}}$ is much greater than the external fluid conductivity $\sigma_{\mathrm{o}}$, we have

$\nabla \varphi_{\mathrm{i}} \cdot \mathbf{n}=O(\alpha)$

and therefore the electrical potential $\varphi_{\mathrm{i}}$ is uniform due to the Laplace's Eq. (2). Furthermore, considering boundary condition (4), it comes

$\varphi_{\mathrm{i}}=0$,

and so

$\varphi_{\mathrm{o}}=0 \quad$ on $S_{\mathrm{d}}$

In its turn, the non-dimensionalized Gauss law (7) becomes $(1+\alpha / \beta) \nabla \varphi_{\mathrm{o}} \cdot \mathbf{n}_{\mathrm{o}}=-q_{\mathrm{s}}$ and since $\alpha \ll \beta$ it comes:

$\varepsilon_{\mathrm{o}} \nabla \varphi_{\mathrm{o}} \cdot \mathbf{n}_{\mathrm{o}}=-q_{\mathrm{s}}$

An important consequence is that calculations are restricted to the external dielectric medium phase. The potential function $\phi$ outside the drop is determined by integration of Laplace's Eq. (2) with a set of boundary Eqs. (3), (8), (9) and (12). The potential $\varphi_{\mathrm{o}}$ knowledge gives all electrical parameters; here the charge acquired by the sphere (Eq. (14)) and as we shall see in the next paragraph the force exerted by the electrical field on the sphere:

$Q=-\varepsilon_{\mathrm{o}} \int_{S_{\mathrm{d}}} \nabla \varphi_{\mathrm{o}} \cdot \mathbf{n}_{\mathrm{o}} \mathrm{d} S$.

\subsection{Mechanical equations}

To the electrical equations we must add the mechanical equations. Since both liquids are incompressible there is no electroresistive force acting on the liquid bulk and at the interface. The electric stress tensor and the hydrodynamic 
stress tensor can respectively be written as:

$\overline{\overline{\mathbf{T}}}^{\mathrm{e}}=\varepsilon \mathbf{E} \mathbf{E}-\frac{1}{2} E^{2} \overline{\overline{\mathbf{I}}}$

and

$\overline{\overline{\mathbf{T}}}=-p \overline{\overline{\mathbf{I}}}+2 \mu \overline{\overline{\mathbf{D}}}$,

where $\mathbf{E E}$ is the diadic product of the electrical field with itself, $\overline{\overline{\mathbf{I}}}$ is the identity tensor, $p$ is the pressure, $\mu$ is the dynamic viscosity, $\overline{\overline{\mathbf{D}}}$ is the deformation rate tensor.

$\overline{\overline{\mathbf{D}}}=\frac{1}{2} \overline{\overline{\left[\nabla \mathbf{u}+(\nabla \mathbf{u})^{+}\right.}}$,

where $\mathbf{u}$ is the possible velocity field and the superscript "+" stands for the transpose.

The tangential component of momentum balance at the drop interface, where $\mathbf{t}$ is the unit vector tangent to the interface, is

$2 \mu[[\mathbf{n} \cdot \overline{\overline{\mathbf{D}}}]] \cdot \mathbf{t}-\left(\nabla_{\mathrm{s}} \gamma\right) \cdot \mathbf{t}=q_{\mathrm{s}} \nabla \varphi \cdot \mathbf{t} \quad$ on $S_{\mathrm{d}}$.

Since the electrical potential inside the drop is uniform, the tangential field is zero, $\nabla \varphi \cdot \mathbf{t}=0$. Moreover, temperature is supposed uniform everywhere, so the surface gradient $\nabla_{\mathrm{s}}$ of the interfacial tension $\gamma$, i.e. the Marangoni stress, is zero. In absence of forcing term at the interface $\overline{\overline{\mathbf{D}}}$ is zero and there is no circulatory motions $\mathbf{u}$ inside and outside the droplet.

The Navier-Stokes equation under hydrostatic conditions is a balance between volume and pressure forces:

$\rho \mathbf{g}-\nabla p=0$.

The Eq. (19) may be integrated, from $z=0$ where $p(0)$ is different for each liquid, to an arbitrary height $z$, and written as:

$\rho \mathbf{g} z-p=p(0)$.

The final drop shape is furnished by the normal component of momentum balance at the drop interface:

$[[\mathbf{n} \cdot \overline{\overline{\mathbf{T}}}]] \cdot \mathbf{n}_{\mathrm{o}}-\gamma \nabla_{\mathrm{s}} \cdot \mathbf{n}_{\mathrm{o}}=-\left[\left[\overline{\overline{\mathbf{T}}}^{\mathrm{e}}\right]\right] \cdot \mathbf{n}_{\mathrm{o}} \quad$ on $S_{\mathrm{d}}$,

where $\nabla_{\mathrm{s}} \cdot \mathbf{n}_{\mathrm{o}}$ is the mean curvature of the interface expressed as a surface divergence. Taking into account Eqs. (15), (16) and (20), this equation becomes:

$\gamma \nabla_{\mathrm{s}} \cdot \mathbf{n}_{\mathrm{o}}=-\left(\rho_{\mathrm{i}}-\rho_{\mathrm{o}}\right) g z+\Delta p+\frac{\varepsilon_{\mathrm{o}}}{2}\left(\nabla \varphi_{\mathrm{o}} \cdot \mathbf{n}_{\mathrm{o}}\right)^{2} \quad$ on $S_{\mathrm{d}}$,

where $\Delta p=p_{\mathrm{i}}(0)-p_{\mathrm{o}}(0)$ is the pressure difference over the electrode between the droplet and the surrounding fluid. Usually, it has been found convenient by most authors to chose the apex as origin and an unknown parameter which is based on the radius of curvature of the apex.

Note that the overall electrical force is simply:

$F=\frac{1}{2} \varepsilon_{\mathrm{o}} \int_{S_{\mathrm{d}}}\left(\nabla \varphi_{\mathrm{o}} \cdot \mathbf{n}_{\mathrm{o}}\right)^{2} \mathbf{z} \cdot \mathbf{n}_{\mathrm{o}} \mathrm{d} S=\int_{S_{\mathrm{d}}} p_{\text {elec }} \mathbf{z} \cdot \mathbf{n}_{\mathrm{o}} \mathrm{d} S$,

where $p_{\text {elec }}$ is the electrostatic pressure on the fluid/fluid interface $S_{\mathrm{d}}$.
Eq. (22) is rewritten by using $a_{0}, \gamma / a_{0}$ and $a_{0} E_{\infty}$ to scale respectively length, pressure and electrical potential:

$\nabla_{\mathrm{s}}^{*} \cdot \mathbf{n}_{\mathrm{o}}=-E \ddot{o} z^{*}+\lambda+\frac{1}{2} B_{\mathrm{e}}\left(\nabla^{*} \varphi_{\mathrm{o}}^{*}\right)^{2}$ on $S_{\mathrm{d}}$.

Superscript stars indicate dimensionless variables. From now on, it will be dropped. Three dimensionless numbers appear: the Eötvos number $E \ddot{o}=\left(\left(\rho_{\mathrm{i}}-\rho_{\mathrm{o}}\right) g a_{0}^{2}\right) / \gamma$, which is also known as the Bond number [11], measures the importance of the gravitational force compared to the surface tension force; the electrical Bond number $B_{\mathrm{e}}=$ $\varepsilon_{0} E_{\infty}^{2} a_{0} / \gamma$ which is the analog using the electrical force instead of the gravitational one; and $\lambda=\Delta p a_{0} / \gamma$ which is the non-dimensional pressure difference [12].

Several definitions of $E \ddot{O}$ and $B_{\mathrm{e}}$ exist depending upon the length scale chosen. The length scale specific to our situation will be the radius of the spherical cap $a$. In this way, alternative dimensionless numbers, denoted by an overbar, can be defined: $E \ddot{o}=\delta^{2} \bar{E} \ddot{o}, B_{\mathrm{e}}=\delta \overline{B_{\mathrm{e}}}$ and $\lambda=\delta \bar{\lambda}$ where $\delta$ is given in Eq. (1).

At order 1, we obtain an obvious condition valid for the undeformable case, showing that the fluid/fluid interface of the droplet is locally spherical:

$2=\bar{\lambda}$.

Generally, for a general deformable case, Eq. (24) is a second-order differential equation; it needs two boundary conditions. The angle between the horizontal plane and the interface is $0^{\circ}$ at the apex and this angle becomes the contact angle $\theta_{\mathrm{s}}$ at the $\operatorname{rim} z=0$. Furthermore, as this equation has an unknown parameter $\bar{\lambda}$ in its second member, an extra condition of constant volume of the drop has to be included. In fact, we shall see that the condition bearing on $\theta_{\mathrm{s}}$ is not appropriate when the electrical field is applied.

\section{Non-wetting conditions}

\subsection{Existing theories}

Non-wetting droplets can be assimilated as perfect spheres of radius $a_{0}$ tangent to the electrode. Lebedev and Skal'skaya [4] integrated the Laplace's Eq. (2) with boundary conditions (3), (8), (9) and (12) in the "tangentsphere coordinates system". Next they calculated, the charge $Q_{0}$ acquired by the sphere, namely the Maxwell's charge, and the electrical force $\mathbf{F}_{0}$ exerted by the electrical field $\mathbf{E}_{\infty}$ on the sphere. One restriction is that the radius $a_{0}$ of this sphere must be negligible compared to the inter plate distance $H$ :

$Q_{0}=4 \pi \varepsilon_{0} E_{\infty} a_{0}^{2} \zeta(2)$,

$F_{0}=4 \pi \varepsilon_{o} E_{\infty}^{2} a_{0}^{2}\left\{\zeta(3)+\frac{1}{6}\right\}$

where the function $\zeta$ is Riemann's Zeta function. The subscript 0 refers to the non-wetting case.

Under the effect of an electric field, the free charge carriers of a perfect conductor are spread over its surface; 
global charge and electric force must therefore be linked to the conductor surface. In expressions (26) and (27), these values are proportional to the sphere's surface area $4 \pi a_{0}^{2}$.

It has been demonstrated that the acquired charge $Q_{0}$, called the Maxwell charge, depends on the sign of the electric field, but the force $F_{0}$ exerted along the axis perpendicular to the electrode always tends to detach the sphere. Indeed, the force being proportional to the square of the applied electric field intensity, it is independent of the field sign. Whatever the sign of the electric field, the acquired charges force the sphere to depart from the electrode. However, the study of Lebedev and Skal'skaya [4] does not explain the exact nature of the electrostatic force $\mathbf{F}$ which is not only the Coulombic force $Q_{0} E_{\infty}$.

The previous approach, based on the use of bispherical coordinates was taken up and confirmed later by Jones [6] using a multipolar approach. Here, the force is divided into one component tending to lift the sphere (Coulombic force $Q_{0} E_{\infty}$ relative to the global charge taken by the sphere) and one attractive component, due to the mutual force of attraction between the sphere and its image in relation to the plate; indeed, the presence of the electrode with uniform potential imposes deformation of the electric field around the sphere.

Consider a droplet heavier than the surrounding dielectric fluid and deposited on a horizontal superhydrophobic electrode. Prior to lift-off, the overall equilibrium condition state that the reaction force $\mathbf{R}$ from the substrate equals the Archimedean force $\left(\rho_{\mathrm{i}}-\rho_{\mathrm{o}}\right) V \mathbf{g}$ corrected by the electrostatic force $\mathbf{F}$ :

$R=\left(\rho_{\mathrm{i}}-\rho_{\mathrm{o}}\right) V g-F$.

Above a certain inter-plate voltage, the electrostatic force will cause the sphere to lift-off. The threshold field $E_{\infty}^{\mathrm{c}}$ for lifting a sphere can be evaluated by writing that $R$ is zero. Then the electrostatic force (Eq. (27)) must equals the weight of the sphere minus buoyancy:

$E_{\infty}^{\mathrm{c}}=\sqrt{\frac{1 / 3\left(\rho_{\mathrm{i}}-\rho_{\mathrm{o}}\right) a_{0} g}{\varepsilon_{\mathrm{o}}\{\zeta(3)+1 / 6\}}}$.

After being lifted from the lower electrode, the electrically charged droplet moves towards the upper electrode. The attraction between the sphere and its image (Fig. 2) decreases as the droplet/electrode distance $z$ increases (Fig. 3).

Davis [13] used bispherical coordinates to solve the general problem of two perfectly conducting, electrically charged spheres at arbitrary spacing in a uniform electric field. To calculate the force, he obtained a series expression for the electric field and then integrated the Maxwell stress tensor over the sphere surface. By imposing a constant charge $Q_{0}$ for calculations, we checked numerically that the force value $F$ exerted on a sphere increases with the distance, from the force value calculated by Lebedev and Skal'skaya [4] at $z \sim 0$ to the Coulombic force $Q_{0} E_{\infty}$ at infinity. Fig. 3 shows the evolution of the two components

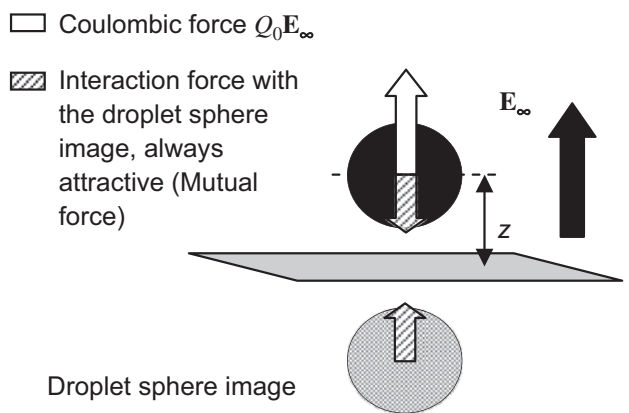

Fig. 2. A conducting sphere after lift-off.

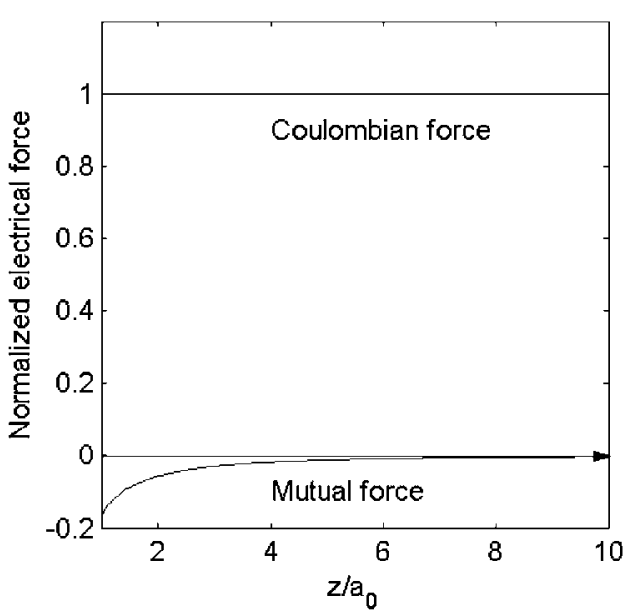

Fig. 3. Conductive sphere subjected to a uniform electric field near a flat electrode. Evolution of the two components of the force is indicated.

of the electric force on a sphere; the curves have been normalized with $Q_{0} E_{\infty}$. The figure shows that the influence of the electrode for the sphere disappears for $z / a_{0}>4$.

Note that the force expression obtained by Davis [13] cannot be evaluated at $z=0$ because the bispherical coordinates are no longer suitable. Lebedev and Skal'skaya's [4] expressions can be recovered as limits of Davis' expressions but the calculations are not straightforward. To our knowledge only Bentwich and Miloh [14] showed how to calculate such a limit.

\subsection{Experimental setup}

The condenser is made of two parallel copper frames with dimensions $50 \times 150 \mathrm{~mm}^{2}$ each (Fig. 4). The experimental cell is placed between the conductive frames and its diameter is $33 \mathrm{~mm}$. The lower frame is surmounted by a hydrophobic carbon nanotubes covered silicon plate (Fig. 5). The electrical connection between the conductive carbon nanotubes covered surface and the copper frame is ensured by a conductive varnish. The upper electrode is coated with a very thin hydrophobic layer of Cytop.

The condenser is filled with a light mineral oil (M-3516 Sigma) of density 0.84 , relative permittivity 2.11 and conductivity $\sigma_{\mathrm{o}}=396 \times 10^{-15} \pm 4 \times 10^{-15} \mathrm{~S} / \mathrm{m}$. In the following 


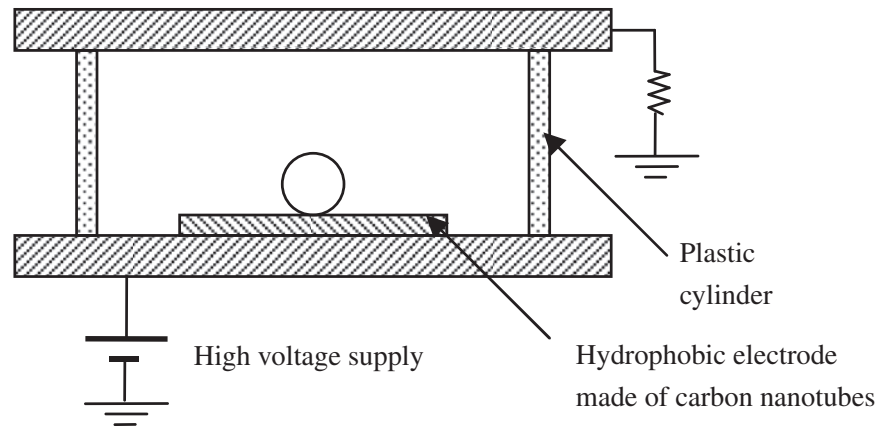

Fig. 4. Experimental setup.

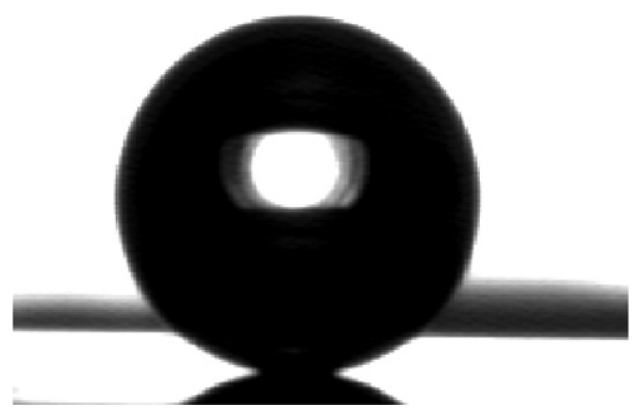

Fig. 5. Water droplet $(\mathrm{NaCl} 0.5 \mathrm{M})$ deposited on a superhydrophobic electrode. The surface hysteresis is negligible, for a slight angle the droplet slides on the surface as a solid ball would do.

experiments, a saturate $\mathrm{NaCl}$ solution $\left(\rho_{\mathrm{i}}=1.184 \times 10^{3} \mathrm{~kg} / \mathrm{m}^{3}\right)$ and a $0.5 \mathrm{M} \mathrm{NaCl}$ solution $\left(\rho_{\mathrm{i}}=1.015 \times 10^{3} \mathrm{~kg} / \mathrm{m}^{3}\right)$ are used to create very conducting droplets of different density.

The lower electrode is connected to a TreK 677B high voltage supply controlled by a Yokogawa FG120 function generator. The upper electrode is connected, through a $1 \mathrm{M} \Omega$ resistance, to the ground.

A step voltage is applied to the condenser for $\varepsilon_{\mathrm{o}} / \sigma_{\mathrm{O}} \sim 45 \mathrm{~s}$. When the electric field is close to the critical one $E_{\infty}^{\mathrm{c}}$, droplets are slightly elongated. The critical electric fields are simply deduced from the lift-off voltage $V_{\infty}^{\mathrm{c}}$ by the relation $E_{\infty}^{\mathrm{c}}=V_{\infty}^{\mathrm{c}} / H$. The critical field $E_{\infty}^{\mathrm{c}}$ is bracketed by an upper electrical field such that a droplet of a given volume takes-off five consecutive times and a lower electrical field such that the same droplet does not takesoff five consecutives times. The experimental results are compared to those computed from Eq. (29).

On Fig. 6, the minimum electrostatic field to lift droplets $E_{\infty}^{\mathrm{c}}$ is plotted as a function of droplets volume. Two series of experiments are shown: one concerns droplets from a $0.5 \mathrm{M} \mathrm{NaCl}$ solution and the other concerns droplets from a $\mathrm{NaCl}$ saturated solution. The theoretical prediction (solid lines) shows an excellent agreement with the experimental threshold. So the theory, valid for solid conducting balls, can be extended to conducting droplets in non-wetting conditions. The slight deformation observed in experiments appears to be no significant here.

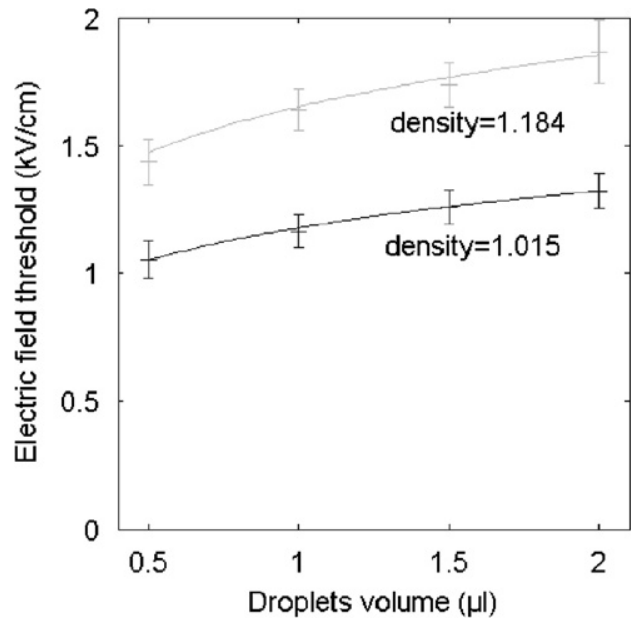

Fig. 6. Experimental and theoretical lift-off field threshold plotted as functions of the droplet volume. The solid line is the theoretical threshold.

\section{Wetting conditions}

\subsection{Adaptation of Le Ny's theory}

The method used by Lebedev and Skal'skaya [4] was generalized to the case of a portion of sphere protruding from a plane electrode by Le Ny [9]. This last work seems to not have gained the same attention as Lebedev and Skal'skaya [4], Félici [5] or Jones [6] works. The charge $Q$ and the force $F$ were given as functions of the protrusion radius $a$ and contact angle $\theta$. To some extent, the work of Le $\mathrm{Ny}$ is close to the problem considered in the present paper: an undeformable wetting droplet (Fig. 7) is similar to a sphere more or less embedded in a plane. Therefore, the charge and force expressions obtained by $\mathrm{Le} \mathrm{Ny}$ [9] can be adapted to our problem with minor changes: in Le Ny's problem the relevant parameters are the protrusion radius $a$ and contact angle $\theta$, while in the problem of a droplet relevant parameters are the volume $V$ and the contact angle $\theta_{\mathrm{s}}$.

Let $a$ be the radius of the spherical cap and $C$ its center. The sphere $(C, a)$ and the plane $(z=0)$ intersect in a circle of radius $c$ and center $O$ (Fig. 7). Le Ny [9] integrated the Laplace's Eq. (2) with boundary conditions (3), (8), (9) and (12) in toroidal coordinates (calculations are described in Appendix A). Next Le Ny [9] gave the expression of $Q\left(\theta_{\mathrm{s}}\right)$ and $F$ as function of $\eta_{\mathrm{s}}=\pi-\theta_{\mathrm{s}}$ and $a$, the sphere radius (or $c$, the contact area radius). Here, these expressions are adapted to describe the charge and force on a droplet since its volume $V$ is known while its radius is not. Besides, the original resulting expressions are plotted for constant droplet's volume $V$.

The total electrical charge $Q\left(\theta_{\mathrm{s}}\right)$ on the droplet is given by the expression:

$$
\begin{aligned}
Q\left(\theta_{\mathrm{s}}\right)= & \left.4 \pi \varepsilon_{\mathrm{o}} E_{\infty} \frac{3 V \sin ^{3}\left(\theta_{\mathrm{s}}\right)}{4 \pi\left(1+2 \sin ^{2}\left(\theta_{\mathrm{s}} / 2\right)\right) \cos ^{4}\left(\left(\pi-\theta_{\mathrm{s}}\right) / 2\right)}\right)^{2 / 3} \\
& \times\left(\frac{1}{\left(\pi-\theta_{\mathrm{s}}\right)^{2}} \frac{\pi^{2}}{6}+\frac{1}{12}\right)
\end{aligned}
$$




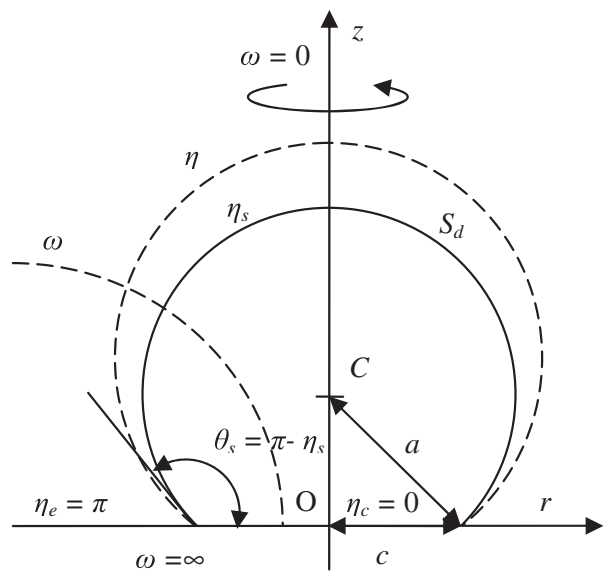

Fig. 7. Droplet wetting a plane electrode with a contact angle $\theta_{\mathrm{s}}$.

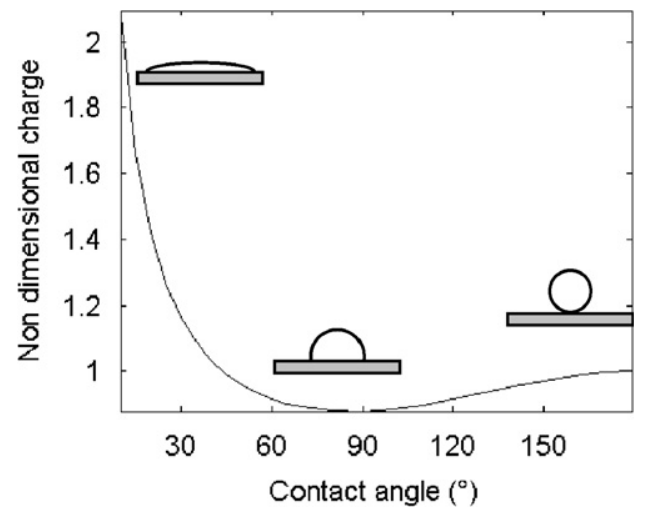

Fig. 8. Charge scaled with $Q_{0}$ and plotted as a function of $\theta_{\mathrm{s}}$ in the range $\left(10^{\circ}, 180^{\circ}\right)$.

and plotted as a function of $\theta_{\mathrm{s}}$ in Fig. 8. On hydrophobic surface, the electrical charge tends towards $Q_{0}$, the Maxwell's charge just found previously, for $\theta_{\mathrm{s}}=180^{\circ}$. On the contrary, when the contact angle becomes very small, the charge increases notably. Indeed, in the case of weak contact angle, the charge is nearly $\varepsilon_{\mathrm{o}} E_{\infty} S_{\mathrm{d}}$ with $S_{\mathrm{d}}$ increasing very fast as $\theta_{\mathrm{s}}$ decreases.

At the fluid/fluid interface, the electrostatic pressure $p_{\text {elec }}=1 / 2 \varepsilon_{\mathrm{o}}\left(\nabla \varphi_{\mathrm{o}} \cdot \mathbf{n}_{\mathrm{o}}\right)^{2}$ is given by the expression:

$$
\begin{aligned}
p_{\text {elec }}= & 4 \varepsilon_{\mathrm{o}} E_{\infty}^{2}\left[\cosh (\omega)-\cos \left(\pi-\theta_{\mathrm{s}}\right)\right]^{3} \frac{\pi^{2}}{2 \sqrt{2}\left(\pi-\theta_{\mathrm{s}}\right)^{3}} \\
& \left.\times \int_{\varpi}^{\infty} \frac{\sinh \left((\lambda / 2)\left(\pi / \pi-\theta_{\mathrm{s}}\right)\right)}{\cosh ^{3}\left((\lambda / 2)\left(\pi / \pi-\theta_{\mathrm{s}}\right)\right)} \frac{\mathrm{d} \lambda}{\sqrt{\cosh (\lambda)-\cosh (\omega)}}\right)^{2}
\end{aligned}
$$

which was simplified using a integral representation of the Mehler function and the familiar properties of Fourier sine transforms [9]. This latter expression, represented in Fig. 9, shows that the pressure is negligible on the interface facing the electrode and increases very fast in the upper part of the droplet.

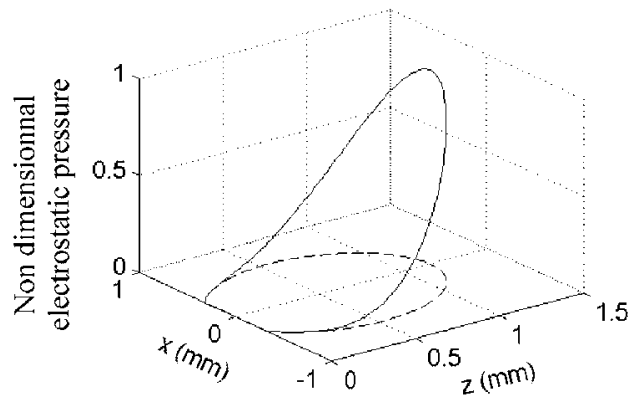

Fig. 9. Electrostatic pressure on the interface of a $1 \mu \mathrm{l}$ droplet lying on an electrode with the contact angle $\theta_{\mathrm{s}}=150^{\circ}$. The pressure is normalized by its maximum at the apex.

The force acting on a droplet is deduced from Eq. (31) using expression (23):

$$
\begin{aligned}
F= & \left.\pi^{3} \varepsilon_{\mathrm{o}} E_{\infty}^{2} \frac{3 V \sin ^{3}\left(\theta_{\mathrm{s}}\right)}{4 \pi\left(1+2 \sin ^{2}\left(\theta_{\mathrm{s}} / 2\right)\right) \cos ^{4}\left(\pi-\theta_{\mathrm{s}} / 2\right)}\right)^{2 / 3} \\
& \times \int_{0}^{\infty}\left(1-\cosh (\omega) \cos \left(\pi-\theta_{\mathrm{s}}\right)\right) \sinh (\omega)\left(\frac{1}{\left(\pi-\theta_{\mathrm{s}}\right)^{3}}\right. \\
& \left.\times \int_{\omega}^{\infty} \frac{\sinh \left((\lambda / 2)\left(\pi / \pi-\theta_{\mathrm{s}}\right)\right)}{\cosh ^{3}\left((\lambda / 2)\left(\pi / \pi-\theta_{\mathrm{s}}\right)\right)} \frac{\mathrm{d} \lambda}{\sqrt{\cosh (\lambda)-\cosh (\omega)}}\right)^{2} \mathrm{~d} \omega .
\end{aligned}
$$

To our knowledge, this complex expression has not been simplified yet. Introducing $h(\theta)$ as a shape function, expression (32) becomes:

$F=h\left(\theta_{\mathrm{s}}\right) F_{0}$

with

$$
\begin{aligned}
h\left(\theta_{\mathrm{s}}\right)= & \frac{\pi^{2}}{4(\zeta(3)+(1 / 6))} \\
& \left.\times \frac{\sin ^{3}\left(\theta_{\mathrm{s}}\right)}{\left(1+2 \sin ^{2}\left(\theta_{\mathrm{s}} / 2\right)\right) \cos ^{4}\left(\pi-\theta_{\mathrm{s}} / 2\right)}\right)^{2 / 3} \\
& \times \int_{0}^{\infty}\left(1-\cosh (\omega) \cos \left(\pi-\theta_{\mathrm{s}}\right)\right) \sinh (\omega) \\
& \times \frac{1}{\left(\pi-\theta_{\mathrm{s}}\right)^{3}} \int_{\omega}^{\infty} \frac{\sinh \left((\lambda / 2)\left(\pi / \pi-\theta_{\mathrm{s}}\right)\right)}{\cosh ^{3}\left((\lambda / 2)\left(\pi / \pi-\theta_{s}\right)\right)} \\
& \left.\frac{\mathrm{d} \lambda}{\sqrt{\cosh (\lambda)-\cosh (\omega)}}\right)^{2} \mathrm{~d} \omega .
\end{aligned}
$$

Fig. 10 shows the result of the numerical evaluation of Eq. (34). It appears that the force presents a minimum at nearly $\theta_{\mathrm{s}}=50^{\circ}$ and increases with the contact angle $\theta_{\mathrm{s}}$ to reach asymptotically the value $F_{0}$ given by Lebedev and Skal'skaya [4]. One would note that in the case of small contact angles, the force increases quickly as $\theta_{\mathrm{s}}$ decreases.

As a consequence, even in the case of negligible capillary forces, the electrical field required to lift a droplet increases with the wetting. 


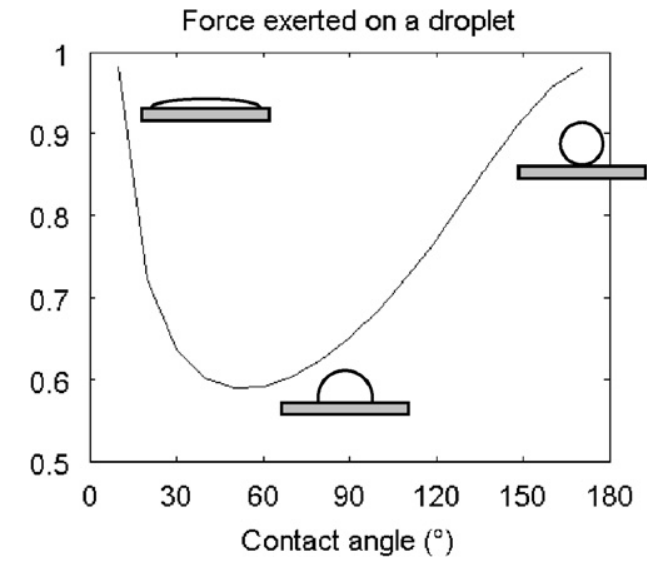

Fig. 10. Electrical force scaled, $h\left(\theta_{\mathrm{s}}\right)$, in the range $\left(10^{\circ}, 170^{\circ}\right)$.

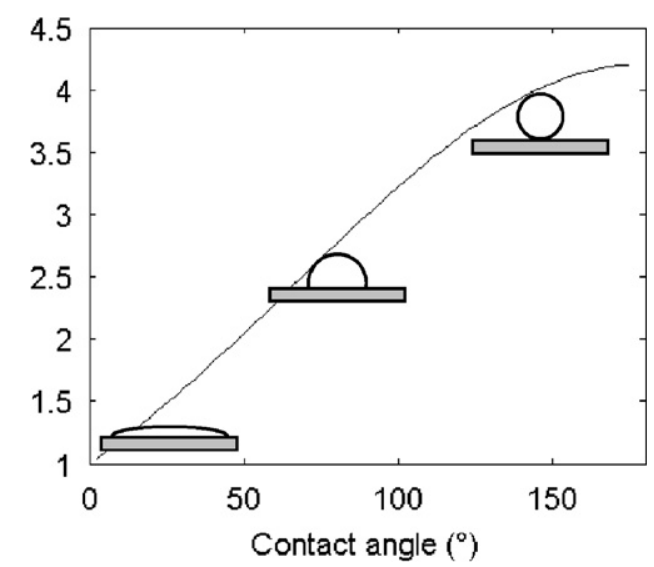

Fig. 11. Electrical field $E_{\mathrm{a}}$ at the apex scaled by $E_{\infty}$ and plotted as a function of $\theta$.

It is also interesting to evaluate the electrical field at the apex:

$E_{\mathrm{a}}=2^{3 / 2} E_{\infty}[1-\cos (\eta)]^{3 / 2} \int_{0}^{\infty} \frac{\tanh (\lambda \pi)}{\sinh (\lambda \eta)} \lambda^{2} \mathrm{~d} \lambda$.

$E_{\mathrm{a}} / E_{\infty}$ is a function of the contact angle $\theta_{\mathrm{s}}$ and varies from 1 for a plane surface to 4.2 for a perfect sphere (Fig. 11). An important consequence is the electrical field amplification as a function of the wetting.

Note that the expressions (30) and (32) given for conducting spherical cap and functions of the contact angle $\theta_{\mathrm{s}}$ must have Eqs. (26) and (27) as limits for vanishingly small $c\left(\theta \rightarrow 180^{\circ}\right)$. The limit calculation, analogue to Bentwich and Miloh [12] calculations, is described in detail in Appendix B.

\subsection{Equilibrium condition on a wetting droplet}

As in the non-wetting case, prior to lift-off, the overall equilibrium condition states that the interaction force $R$ from the substrate equals the Archimedean force $\left(\rho_{\mathrm{i}}-\rho_{\mathrm{o}}\right) V g$ corrected by the electrostatic force $F$. Eq. (28) is still valid but the interaction force $R$ from the substrate is quite different since it involves the adhesion of the drop to the electrode which can be expressed as $2 \pi c \gamma \sin (\theta)$ corrected by the force applied on the contact area $\pi c^{2}$ arising from the pressure difference $\Delta p$. This latter force $\pi \Delta p c^{2}$ is overlooked by many authors:

$R=\pi \Delta p c^{2}-2 \pi c \gamma \sin (\theta)$.

Finally, the overall equilibrium condition is

$\pi \Delta p c^{2}-2 \pi c \gamma \sin (\theta)=\left(\rho_{\mathrm{i}}-\rho_{\mathrm{o}}\right) V g-F$,

whether the droplet is undeformable or not. If the droplet is undeformable then the value of $F$ is given by Eq. (32).

As previously, the threshold field $E_{\infty}^{\mathrm{c}}$ for lifting a sphere should be evaluated by writing that $R$ is zero. Unfortunately, if we maintain the undeformability assumption there is no way to understand how $c$ defined in Eq. (36) should be reduced to zero. To get $E_{\infty}^{\mathrm{c}}$, the Eq. (37) is of no help.

Furthermore, this equation affords no extra information for a strictly undeformable droplet $\left(E \ddot{o}=0, B_{\mathrm{e}}=0\right)$. Let us restate it in a dimensionless form:

$\pi \frac{c}{a_{0}}\left(\frac{\Delta p}{\gamma} c-2 \sin (\theta)\right)=\frac{4}{3} \pi\left(E \ddot{o}+B_{\mathrm{e}} F^{*}\right)$,

where $F=\varepsilon_{\mathrm{o}} E_{\infty}^{2} a_{0}^{2} F^{*}$. The $O(1)$ order for $E \ddot{o} \ll 1$ and $B_{\mathrm{e}} \ll 1$ becomes:

$\Delta p=\frac{2 \gamma \sin (\theta)}{c}=\frac{2 \gamma}{a}$

which has already been obtained (Eq. (25)).

For instance, consider an experiment (Fig. 12) on a water droplet $(\mathrm{NaCl} 0.5 \mathrm{M})$ whose initial contact angle is about $162^{\circ}$. The Eötvos number 0.01 is very low so the gravity forces can be neglected. The voltage applied to the condenser is increased from $0 \mathrm{~V}$ to $V_{\infty}^{\mathrm{c}}$ the "lift-off voltage". At lift-off, the electrical field intensity is $1.49 \mathrm{kV} / \mathrm{cm}$. Considering the electrical field amplification on the upper part of the cap (Eq. (35)), this leads to an electric Bond number of 0.07 at the apex which is more important than the Eötvos number. For this Bond number value, Fig. 12 shows that the electrical forces begin to compete with surface tension forces.

In conclusion as far as lift-off conditions are concerned, the undeformability assumption must be relaxed. We have to introduce a physical mechanism explaining why the reaction or more precisely the interaction forces vanishes. One mechanism observed by Khayari et al. [3] is the breakup process for drops having some small contact angle and a large contact area. Excluding these situations, we will see in a following paper that deformation, even slight, may let the contact angle vary and reach some critical condition where the contact area is swept away so, in Eq. (36), $R$ tends to zero as $c$ reduces suddenly.

\section{Conclusion}

A uniform electric field exerts a lifting force on a conductive droplet immersed in a dielectric fluid and in contact with an electrode. On one hand, the extensively 

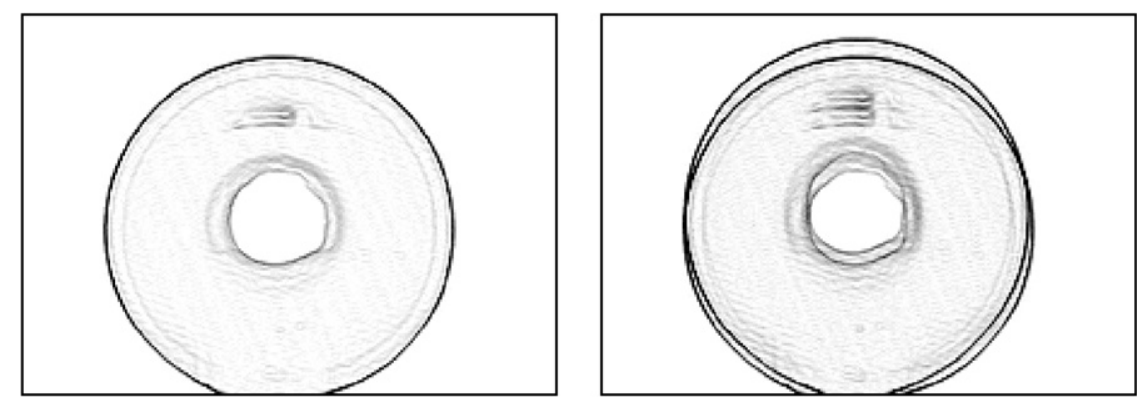

Fig. 12. Water droplet $(\mathrm{NaCl} 0.5 \mathrm{M})$ of $1 \mu 1$ deposited on an electrode covered by a thin layer of Cytop and subjected to a lifting electrical force. The electrical field intensity is increased slowly and reached $1.49 \mathrm{kV} / \mathrm{cm}$ at lift-off. On the left, the electrical field is zero. On the right, the droplet is deformed by the electrical force; the deformed droplet shape is superposed to the undeformed droplet shape.

studied case of solid spheres was transposed to droplets by using a conducting superhydrophobic electrode. In such non-wetting conditions, the lift-off criterion matches the experimental results. On the other hand, the case of wetting droplets was investigated. By restricting the study to an undeformable spherical cap, Le Ny's calculations were adapted to the problem of a droplet wetting an electrode. It appears that the electrical field is amplified at the droplet apex. The amplification factor depends on the contact angle and reaches a maximum of 4.2 in the non-wetting case. It was also shown that the charge acquired by a droplet presents a minimum at $90^{\circ}$. From this minimum, the charge increases very fast as the contact angle tends to the total wetting situation and increases asymptotically as the contact angle varies up to $180^{\circ}$. In the latter case, the charge reaches asymptotically the value predicted by Lebedev and Skal'skaya. But it was also shown experimentally that such a droplet is likely to be deformed when subjected to an electrical field and the contact angle changes. Even in weakly wetting situations $\left(\theta \sim 160^{\circ}\right)$, the droplets deformation should be taken into account to estimate the lift-off criterion.

\section{Acknowledgments}

We would like to thank Claude Chabrol for helping out with the experimental devices and setup; Marc Plissonnier for providing us carbon nanotubes covered electrodes and, finally, Nelly Bonifaci (CNRS Electrostatic and Dielectric Environments Laboratory) for the permittivity and conductivity measurements made on the mineral oil used in our experiments.

This research is supported by the French Research Ministry on the Recherche Technologique de Base program (RTB).

\section{Appendix A}

By introducing toroidal coordinates $(\omega, \eta)$, presented in Fig. 7, Laplace's Eq. (2) becomes:

$$
\begin{aligned}
& \left(\frac{\partial}{\partial \omega}\left(\frac{\sinh \omega}{\cosh \omega-\cos \eta} \frac{\partial}{\partial \omega}\right)\right. \\
& \left.+\frac{\partial}{\partial \eta}\left(\frac{\sinh \omega}{\cosh \omega-\cos \eta} \frac{\partial}{\partial \eta}\right)\right) \varphi=0
\end{aligned}
$$

and boundary conditions (3), (8), (9) and (12) becomes, respectively, Eqs. (A.2), (A.3), (A.4) and (A.5):

$\left.\varphi\right|_{\eta=0}=0 \quad$ on $S_{\mathrm{e}}$

$\left.\varphi\right|_{\eta=\eta_{\mathrm{s}}}=0 \quad$ on $S_{\mathrm{d}}$,

$\varphi(0, \eta)$ is finite on the vertical axis $\mathrm{Oz}$,

$\lim _{z \rightarrow \infty} \varphi=-E_{\infty} c \frac{\sin \eta}{\cosh \omega-\cos \eta}$,

where $\eta_{\mathrm{s}}$ is the value of the $\eta$ coordinate corresponding to the surface $S_{\mathrm{d}}$ of the spherical cap and defined by

$\sin \eta_{\mathrm{s}}=\frac{c}{a}$,

Using the linear property of Laplace's Eq. (2), condition (A.5) leads to:

$\varphi=\varphi_{\mathrm{s}}-E_{\infty} c \frac{\sin \eta}{\cosh \omega-\cos \eta}$,

where $\varphi_{\mathrm{s}}$ satisfy Eq. (A.1) with respect to conditions (A.2)-(A.4). Substituting $\varphi_{\mathrm{p}}(\omega, \eta)=(\cosh \omega-\cos \eta)^{1 / 2} f(\omega) g(\eta)$, it comes:

$g(\eta)=A \sinh (\eta)+B \cosh (\eta)$,

$f(\omega)=C P_{\text {in }-1 / 2}(\cosh (\omega))+D Q_{\text {in }-1 / 2}(\cosh (\omega))$,

where $P_{\text {in }-1 / 2}(\cosh (\omega))$ and $Q_{\text {in-1/2 }}(\cosh (\omega))$ are Legendre's functions of the first and second kind; $A, B, C$, and $D$ are unknown coefficients which are defined by the conditions (A.2)-(A.4).

First of all, condition (A.4) leads to $D=0$ since the Legendre's function $Q_{\mathrm{in}-1 / 2}(\cosh (\omega))$ is singular on the axis Oz. Afterwards, condition (A.2) imposes $B=0$. The potential $\phi$ expression becomes then:

$$
\begin{aligned}
\varphi(\omega, \eta)= & \sqrt{\cosh (\omega)-\cos (\eta)} \int_{0}^{\infty} A_{\lambda} C_{\lambda} \sinh (\lambda \eta) \\
& \times K_{\lambda}(\cosh (\omega)) \mathrm{d} \lambda-E_{\infty} c \\
& \times \frac{\sin (\eta)}{\cosh (\omega)-\cos (\eta)} .
\end{aligned}
$$


To finish with, the unknown coefficient $A_{\lambda} C_{\lambda}$ is determined by using condition (A.3) with Mehler's transforms. After some calculations, it comes:

$\varphi(\omega, \eta)= \begin{cases}2^{3 / 2} c E_{\infty} \sqrt{\cosh (\omega)-\cos (\eta)} & \\ \int_{0}^{\infty} \frac{\sinh \left(\lambda\left(\eta-\eta_{\mathrm{s}}\right)\right)}{\sinh \left(\lambda \eta_{\mathrm{s}}\right)} \tanh (\lambda \pi) \lambda K_{\lambda}(\cosh (\omega)) \mathrm{d} \lambda, & \eta_{\mathrm{s}}<\eta<\pi \\ 0, & 0<\eta<\eta_{\mathrm{s}}\end{cases}$

where $K_{\lambda}(\cosh (\omega))=P_{\mathrm{i} \lambda-1 / 2}(\cosh (\omega))$ is sometimes called the conical function or Mehler function.

The charge $Q$ and the force $F$ are, respectively, calculated from Eq. (A.9) with formula (14) and (23).

\section{Appendix B}

The expressions obtained in Section 4 hold when the droplet wets a plate. It is possible to define a sequence of solutions for a spherical cap of fixed radius $a_{0}$ with the contact area radius $c$ becoming gradually smaller. Since the geometry is completely determined in terms of the ratio $\varepsilon=c / a_{0}$, the solution for the tangent sphere is the limit for vanishingly small $\varepsilon$.

The toroidal coordinates $(\omega, \eta)$ are related to the cylindrical coordinates $(\rho, z)$ by

$\omega+\mathrm{i} \eta=2 \operatorname{coth}(\rho-\mathrm{i} z)$.

This limit process is first employed is defining a new set of independent variable $(\mu, v)$. For cylindrical coordinates $(\bar{\rho}, \bar{z})$ which are scaled with $a_{0}$ as the characteristic length, Eq. (B.1) reduces to

$\omega+\mathrm{i} \eta \underset{\varepsilon \rightarrow 0}{\approx} 2 \varepsilon \frac{1}{\bar{\rho}-\mathrm{i} \bar{z}}$.

Therefore, the sphere-tangent coordinates $(\bar{\mu}, \bar{v})$, which are scaled with $v_{0}=1 / 2 a_{0}$ as the characteristic length, are related to $(\omega, \eta)$ by

$(\bar{\mu}, \bar{v})=\frac{\omega, \eta}{\varepsilon}$.

We now trace the behavior of the various components in the expression for the electric potential in the limiting case of $\varepsilon \sim 0$. The factor involving half power can be approximated; thus

$\sqrt{\cosh (\omega)-\cos (\eta)} \underset{\varepsilon \rightarrow 0}{\approx} \frac{\varepsilon}{\sqrt{2}} \sqrt{\bar{\mu}^{2}+\bar{v}^{2}}$,

where $(\bar{\mu}, \bar{v})$ are assumed constant as $\varepsilon$ is decreased. Next, the equation governing Mehler functions $K_{n}$ reduces to an equation governing Bessel functions:

$$
\begin{aligned}
& \left(\frac{\partial^{2}}{\partial \omega^{2}}+\operatorname{coth}(\omega) \frac{\partial}{\partial \omega}+\left(n^{2}+\frac{1}{4}\right)\right) K_{n}(\cosh (\omega)) \\
& \underset{\varepsilon \rightarrow 0}{\approx} \frac{1}{\varepsilon^{2}}\left(\frac{\partial^{2}}{\partial \bar{\mu}^{2}}+\frac{1}{\bar{\mu}} \frac{\partial}{\partial \bar{\mu}}+\varepsilon^{2}\left(n^{2}+\frac{1}{4}\right)\right) K_{n}(\cosh (\omega))=0,
\end{aligned}
$$

so

$\left.K_{n}(\cosh (\omega)) \underset{\varepsilon \rightarrow 0}{\approx} J_{0} \bar{\mu} \varepsilon \sqrt{\left(n^{2}+\frac{1}{4}\right)}\right)$.

The potential $\phi$ expression given by Le $\mathrm{Ny}$ [9] becomes:

$$
\begin{aligned}
\varphi \underset{\varepsilon \rightarrow 0}{\approx} 2 E c \varepsilon \sqrt{\bar{\mu}^{2}+\bar{v}^{2}} \int_{0}^{\infty} \frac{\sinh (\lambda \varepsilon(\bar{v}-1))}{\sinh (\lambda \varepsilon)} \\
\quad \times \tanh (\lambda \pi) \lambda J_{0}\left(\bar{\mu} \varepsilon \sqrt{\lambda^{2}+\frac{1}{4}}\right) \mathrm{d} \lambda .
\end{aligned}
$$

Introducing a new variable $q=\lambda \varepsilon$, it comes:

$$
\begin{aligned}
\underset{\varepsilon \rightarrow 0}{\approx \approx} 2 E a_{0} \sqrt{\bar{\mu}^{2}+\bar{v}^{2}} \int_{0}^{\infty} \frac{\sinh (q(\bar{v}-1))}{\sinh (q)} \\
\times \tanh \left(\pi \frac{q}{\varepsilon}\right) q J_{0}\left(\bar{\mu} \sqrt{q^{2}+\frac{\varepsilon^{2}}{4}}\right) \mathrm{d} q
\end{aligned}
$$

and

$\varphi \underset{\varepsilon \rightarrow 0}{\approx} E \frac{1}{v_{0}^{2}} \sqrt{\mu^{2}+v^{2}} \int_{0}^{\infty} \frac{\sinh \left(\left(v-v_{0}\right)\left(q / v_{0}\right)\right)}{\sinh \left(v_{0}\left(q / v_{0}\right)\right)} q J_{0}\left(\mu \frac{q}{v_{0}}\right) \mathrm{d} q$.

Finally, introducing $q^{\prime}=q / v_{0}$, the potential reduces to

$\varphi \underset{c \rightarrow 0}{\approx} E \sqrt{\mu^{2}+v^{2}} \int_{0}^{\infty} \frac{\sinh \left(q^{\prime}\left(v-v_{0}\right)\right)}{\sinh \left(q^{\prime} v_{0}\right)} q^{\prime} J_{0}\left(\mu q^{\prime}\right) \mathrm{d} q^{\prime}$

which is the expression given by Lebedev and Skal'skaya [4].

\section{References}

[1] S. Birlasekaran, The measurement of charge on single particles in transformer oil, IEEE Trans. Electr. Insulat. (1991) 1094-1103.

[2] R. Tobazéon, Behaviour of spherical and cylindrical particles in an insulating liquid subjected to a DC uniform field, in: Proceedings of the 11th ICDL, 1993, pp. 415-420.

[3] A. Khayari, A.T. Pérez, F.J. Garcia, A. Castellanos, Dynamics and deformation of a drop in a DC electric field, IEEE Electr. Insulat. Dielectric Phenom., USA, Albuquerque (2003) 682-685.

[4] N.N. Lebedev, I.P. Skal'skaya, Force acting on a conducting sphere in the field of a parallel plate condenser, Soviet Phys.-Tech. Phys. 7 (1962) 268-270.

[5] N.-J. Félici, Forces et charges de petits objets en contact avec une electrode affectée d'un champ électrique, Rev. Gen. Electr. 75 (1966) 1145-1159

[6] T.B. Jones, Electromechanics of Particles, Cambridge University Press, New York, 1995, pp. 183-185.

[7] K.K.S. Lau, J. Bico, K.B.K. Teo, M. Chhowalla, G.A.J. Amaratunga, W.I. Milne, G.H. McKinley, K.K. Gleason, Superhydrophobic carbon nanotube forests, Nano Lett. 3 (2003) 1701-1705.

[8] R. Tobazéon, Charges and discharges of conducting particles in a plane oil gap subjected to a DC voltage, in: Proceedings of the 11th ICDL, 1996, pp. 309-312.

[9] R. Le Ny, The electrostatic problem of a portion of sphere protruding from a plane electrode in an electric field, J. Phys. A: Math. Gen. 14 (1981) 945-955.

[10] E.B. Dussan, The moving contact line, in: Proceedings of a Symposium on Waves on Fluid Interfaces, Academic Press, New York, 1983, pp. 303-324. 
[11] S.S. Sadhal, P.S. Ayyaswamy, J.N. Chung, Transport Phenomena with Drops and Bubbles, Springer, New York, 1997, p. 520 .

[12] P. Concus, Static menisci in a vertical right circular cylinder, J. Fluid. Mech. 34 (1968) 481-495.
[13] M.H. Davis, Two charged spherical conductors in a uniform electric field: forces and field strength, Quart. J. Mech. Appl. Math. 17 (1964) 499-511.

[14] M. Bentwich, T. Miloh, On the exact solution for the two-sphere problem in axisymmetrical potential flow, J. Appl. Mech. 45 (1978) 463-468. 